\title{
Der Isishymnus E.r4 aus dem Isistempel von Assuan
}

The initial part of a hymn to Isis as preserved in her temple in Assuan is presented, together with a parallel from Dendera. Brugsch first identified this parallel, but it apparently received no subsequent attention. The version from Dendera helps to reconstruct the first column of the Assuan text. The implications of this re-discovered parallel for the text's history and its interpretation are briefly discussed.

Der Hymnus an Isis, der sich auf der nördlichen Laibung der Tür zum Sanktuar des Isistempels von Assuan befindet, wurde bereits im I9. Jahrhundert veröffentlicht. ${ }^{\text {I }}$ I 978 bot dann Bresciani einen Übersetzungsvorschlag, der jedoch insbesondere im schlecht erhaltenen Anfang erhebliches Verbesserungspotential birgt. ${ }^{2}$ Das liegt daran, daß Bresciani eine Erkenntnis nicht bewußt war, die bereits I $88_{3}$ Brugsch gemacht hatte und auch in seiner Publikation durch Juxtaposition mit einer Parallele in Dendera visualisierte: Ein SothisIsis-Hymnus in Dendera, der inzwischen in Dend. XV, 25, 7-26, 14 erschienen ist (D), ist eine Parallele zum Isis-Hymnus von Assuan (A), die nur an einzelnen Stellen geringfügig, teilweise nur in Graphien abweicht. In Brugschs Thesaurus ist diese Gegenüberstellung durch das Layout nicht sehr deutlich, sondern durch eine Durchzählung der Kolumnen so

${ }^{1}$ H. Brugsch, Thesaurus inscriptionum Aegyptiacarum: Altägyptische Inschriften gesammelt, verglichen, übertragen, erklärt und autographiert von Heinrich Brugsch (Leipzig, 1883-1891), 14; J. de Morgan, U. Bouriant, G. Legrain, G. Jéquier, und A. Barsanti, Catalogue des monuments et inscriptions de l'Egypte antique, I/I: De la frontière de Nubie à Kom Ombo (Vienna, [1894]), 55.

${ }^{2}$ E. Bresciani, 'Il tempio tolemaico di Isi', in E. Bresciani und S. Pernigotti (Hg.), Assuan (Pisa, r 978 ), г $0_{3}-5$, Taf. 22. 
gestaltet, daß ein flüchtiger Leser meinen könnte, es sei nur die D-Fassung wiedergegeben. ${ }^{3}$ Tatsächlich kennzeichnet aber ein 'A' in Brugschs Kolumne 4 den Beginn der A-Rezension. Offenbar scheint wie Bresciani niemand mehr nach ihr Brugschs Thesaurus konsultiert zu haben, denn weder erwähnen Leitz noch v. Lieven die D-Version als Parallele in einer Übersetzung der D-Version ${ }^{4}$ bzw. anläßlich einer Bemerkung zu A, ${ }^{5}$ noch ergänzt Preys seine Übersetzung von A nach D, obwohl die Reste in A eindeutig die Parallelisierung indizieren. ${ }^{6}$ Kockelmann schließlich spricht lediglich von einem 'ähnlichen Hymnus', statt einer Parallele. ${ }^{7}$ Es scheint also an der Zeit, wieder an die Parallelität der zwei Texte zu erinnern, zumal sich die Fehlstellen in A durch D ergänzen lassen. Zunächst die erste Kolumne von A in Synopse mit D:
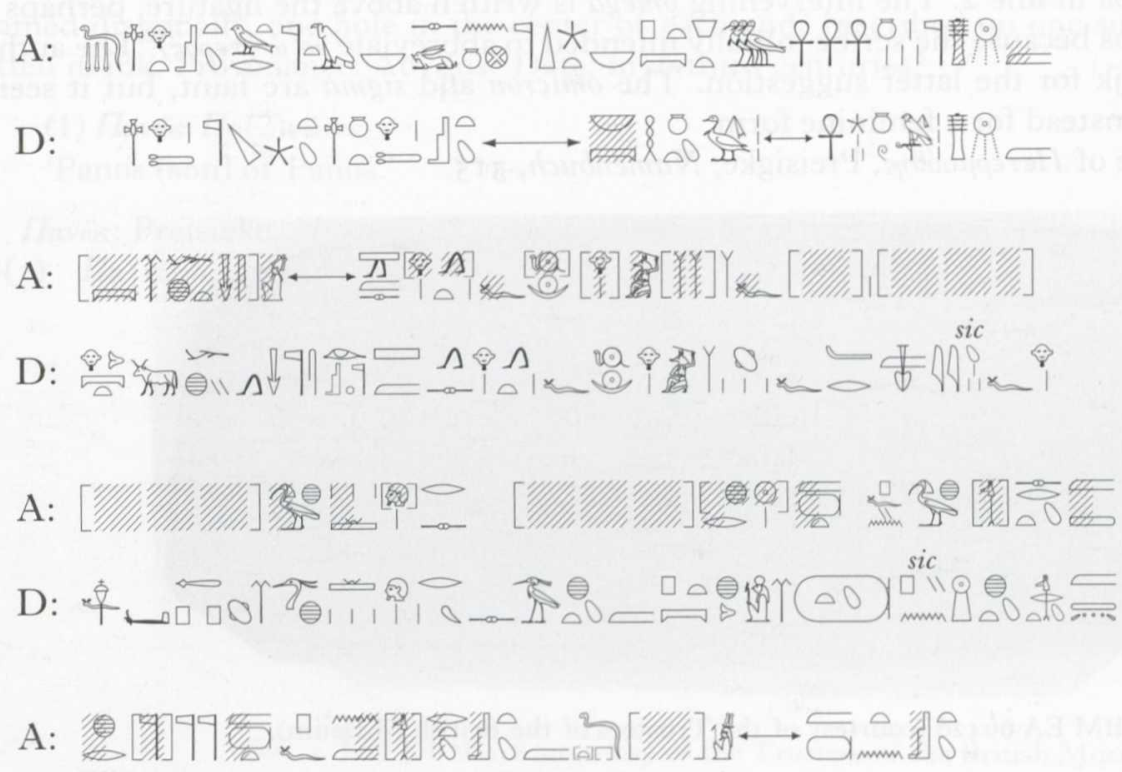

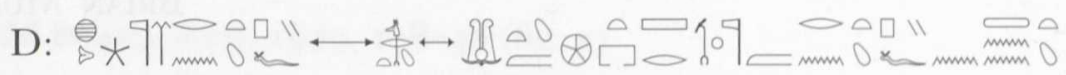

Das Wissen um die Entsprechung von A und D befördert in erster Linie das Verständnis der ersten Kolumne von A. Um diese Miszelle nicht über Gebühr in die Länge zu ziehen, wird deshalb auf eine hieroglyphische Synopse des gesamten Hymnus verzichtet, auch wenn die zweite Kolumne ebenfalls parallel läuft. Doch ist A hier nicht beschädigt und insofern in seiner Lesung unproblematisch. Es sei jedoch eine vollständige Transliteration und Übersetzung mit einem knappen philologischen Kommentar des gesamten Hymnus und des in A sich anschließenden Liedes zum Sistrumspiel geboten, um einen Gesamteindruck der Inschrift zu geben. Die nicht geschriebenen femininen Endung sind stets als ergänzt gekennzeichnet worden, auch wenn der Wegfall derselben für Inschriften dieser Zeit nicht außergewöhnlich ist, weil eine solche Visualisierung für die Argumentation in der Anmerkung ( $t-\mathrm{I})-(\mathrm{t}-2)$ wichtig sein wird.

\footnotetext{
3 So sogar einer der beiden anonymen Gutachter des $\mathscr{Y} E A$ noch nach Lektüre einer Vorversion dieser Miszelle. Leider ist dem nicht so, denn ich mußte feststellen, daß Brugsch bereits vor mir die Parallelität erkannt hatte.

${ }^{4}$ C. Leitz, 'Die Sternbilder auf dem rechteckigen und runden Tierkreis von Dendera', $S A K K_{34}(2006), 2^{28} 5^{-}$ 318 .

5 A. von Lieven, Der Himmel über Esna: Eine Fallstudie zur religiösen Astronomie in Ägypten (ÄA 64; Wiesbaden, 2000), 23.

${ }^{6}$ R. Preys, in Thesaurus Linguae Aegyptiae < http://aaew.bbaw.de/tla/>, s.v. Gliederungshierarchie der Objekte und Texte $>$ Leuven Online Index of Ptolemaic and Roman Hieroglyphic Texts, Katholieke Universiteit Leuven $>$ Assouan $>$ porte du sanctuaire $>$ épaisseurs $>$ montant nord $>$ hymne $>j n d h r$ Isis. Überprüft am ro. September 2012.

7 H. Kockelmann, Praising the Goddess: A Comparative and Annotated Re-Edition of Six Demotic Hymns and Praises to Isis (AfP Beiheft I5; Berlin, 2008), Anm. 2 r6.
} 


\section{Kol. I}

$\underline{d} d$ mdw.w ind $-h r=t$

ss.t wr.t mw.t ntr $n b$ 〈.t) Swn.$t\rangle$

Spd.t nb<.t) p.t hnnw.t b3.w 'nh.w n.w ntr.w

$p s \underline{d}\left\langle. t{ }^{\ulcorner} m^{\urcorner}[h r . t m-h t s n=s]{ }^{\ulcorner} W \operatorname{sir} \check{s} m=s^{\urcorner}\left[h r^{\mathrm{a}}\right.\right.$ $n m t]^{\top} . t=f r c-n b^{\top}$

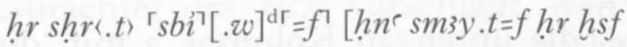

$\left.{ }^{3} p p m\right] 3 h . w^{\mathrm{e}} d p^{\mathrm{f}\urcorner} r_{3}=s$

$[3 h] . t[m p . t h]^{\ulcorner} r^{\urcorner \mathrm{g}}[R \tau]^{\mathrm{h}} m r n=t-p f y n^{\ulcorner} 3 h . t^{\urcorner}$

$[w] s^{\Gamma} r^{\top} . t m t 3 h r{ }^{\Gamma} n \underline{t} r . w m r n=t-p f n-m^{j]}$

$[w] s r . t$

$\Gamma_{3 s . t} m^{\urcorner}$dww.t $[h r] W \operatorname{sir} m r n=t\langle-p f y\rangle$ ss.t

\section{Kol. 2}

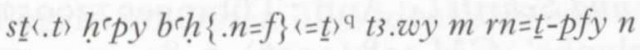
Spd.t

ink $=t-s(w) r$ siwor sh.t $m r n=t-p f y$ enk.t

dis.t) $h p(r)$ wnn.t-nb<.t) $m t 3<n h=s n$ im $=t m$ $r n=t-p f y$ enh.t

skd.t $m$ t3 hr ssḥ wbn.t ḥr isb.t.t n.t p.t

shtp.t $m$ enh hr imn.t.t $r \mathrm{c}-n b$

$n h m=\underline{t}$ nsw-bit Ptwl $\{r\}(m) y s$ s $n h \underline{d}$ d.t mry $3 s . t$ $m-\ulcorner$ h.t-nb<.t) dwus.t) $m-<$ hry.w

\section{Kol. $3^{8}$}

irì.n=i) š̌š.t $m h \grave{h r}=t \quad n f r$

3s.t wr.t mw.t nter nb.t Swn<.t)

ir.t Re iwt.t sn-nw<.t $=s m$ p.t $t 3$

wr<.t) mrw.t ḥnw.t ḥm.wt mhlu.t) p.t $t 3 m$ $n f r . w=s$

$m w . t-n \underline{t} r \quad n<. t) k 3 n h t$ ḥm.t-nsw $n$ Wn-nfr

šps.t ${ }^{v}$ З.$t m$ ḥw.t-sr wै.t $m$ ḥw.t-bnw

$n b$ ct) nmt.t $m$ wis $n$ ḥh iriu.t) shr.w $m$ $d p\langle. t\rangle-n \underline{t} r$

ntr.t 3.t hnt Hw.t-bit ḥnw.t $m$ T3-enh
Worte zu sprechen: Gegrüßt seist du, Isis, die Große, Gottesmutter, Herrin von Syene, Sothis, Herrin des Himmels, Herrscherin der lebendigen Bau der Götter,

die am Himmel erstrahlt ${ }^{\mathrm{b}}$ [hinter ihrem Bruder] Osiris, wenn sie [auf] seinen [Bahnen] geht jeden Tag, ${ }^{c}$ beim Niederwerfen seiner Feind[e zusammen mit seiner Bande, beim Vertreiben des Apophis durch ihre Ach-Sprüche auf] ihrem Mund

[Wirkungsvoll]e [am Himmel vo]r [Re] in diesem deinem Namen einer Wirkungsvollen, ${ }^{i}$

[St] arke in der Erde ${ }^{\mathrm{k}}$ vor den Göttern ${ }^{1}$ in diesem deinen Namen einer [St] arken, ${ }^{\mathrm{m}}$

Isis in der Unterwelt [vor] Osiris ${ }^{\mathrm{n}}$ in deinem Namen in $<$ diesem $>{ }^{\circ}$ deinem Namen Isis, ${ }^{\mathrm{p}}$

die die Überschwemmung ergießt, damit du die Beiden Länder überflutest in diesem deinen Namen Sothis

und du sie (= die Überschwemmung) einleitest, ${ }^{\mathrm{r}}$ um das Feld zu befruchten in diesem deinem Namen der Anukis, ${ }^{\mathrm{s}}$

die, die alles, was ist, entstehen läßt, was auf/in der Erde ist, damit sie leben durch dich in diesem deinem Namen der Lebenden, ${ }^{\mathrm{t}-\mathrm{I}}$

die hinter Orion reist, die im Osten des Himmels aufgeht, ${ }^{\mathrm{t}-2}$

indem sie lebend untergeht im Westen jeden Tag. ${ }^{u}$

Mögest du den König von Ober- und Unterägypten Ptolemaios, der ewig lebe, geliebt von Isis, vor jedem Unheil und vor Aufrührern erretten.

$<$ Ich $>$ spiele das Sistrum vor deinem vollkommenen Angesicht,

Isis, du Große, Gottesmutter, Herrin von Syene,

Auge des Re, deren Gleiche es nicht gibt am Himmel und auf der Erde,

an Liebe Große, Fürstin der Frauen, die Himmel und Erde erfüllt mit ihrer Vollkommenheit,

Gottesmutter des starken Stiers, Königsgemahlin des Wennefer,

Edle, große Herrin im Beamtenhaus, Einzige im Phönixhaus,

Herrin über den Gang ${ }^{\text {w }}$ in der Barke der Millionen, die die Pläne macht in der Gottesbarke,

die große Göttin im Haus der Biene ${ }^{x}$, die Herrin von Taanch, ${ }^{\mathrm{y}}$

${ }^{8}$ Parallelen zu dieser Kolumne mit einer Synopse der Textzeugen: J. F. Quack, 'Ein Standardhymnus zum Sistrumspiel auf einem demotischen Ostrakon (Ostrakon Corteggiani D I)', Enchoria 27 (2001), I I0 und II 7I9. 
hạk.t $m$ W3s.t nb<.t) Išrw

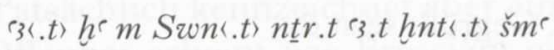
hnww.t mhwo
Herrscherin von Theben, Herrin von Ascheru, an Erscheinung Große in Syene, große Göttin, Erste von Oberägypten, Herrscherin von Unterägypten.

\section{Anmerkungen:}

a Determinativstrich zu $h r$ ist noch erhalten.

b Wegen D ist 'che illumini le due terre [ ] ogni giorno' ${ }^{9}$ nicht richtig.

c Es handelt sich hierbei um eine mythische Aussage, die unten, in Kolumne 2 mit 'die hinter Orion reist' nochmals astronomisch ausgedeutet wiederholt wird.

d Eines von wohl ehemals drei Y ist noch erhalten.

e Die Zeichenreste bei Bresciani vor den Pluralstrichen, von denen nur die letzten zwei erhalten sind, sind vielleicht die Reste eines $3 h$-Vogels. Davor sind weitere Zeichenreste bei Bresciani angegeben, die ich nicht eindeutig zuordnen kann.

f Determinativstrich zu $d p$ noch erhalten. Zur offenbar noch nicht allgemein bekannten Lesung $d p$ für das Zeichen Gardiner Dr siehe D. Werning, 'The Sound Values of the Signs GARDINER Di (Head) and T8 (Dagger)', LingAeg I2 (2004), I 83-204; W. Schenkel, Tübinger Einführung in die klassisch-ägyptische Sprache und Schrift (4. Aufl.; Tübingen, 2005), 58; C. Peust, 'Nochmals zur Lesung der Kopf-Hieroglyphe', GM 208 (2006), 7-8.

g Vor dem $r$ ist bei Bresciani der Rest eines Determinativstrichs zu sehen, den ich nicht zuordnen kann.

h Der Determinativstrich zur Sonnenscheibe ist noch erhalten.

i '[ ] in quel tuo nome di Figlia di Ra, (...)' ${ }^{\text {Io }}$ ist angesichts der D-Parallele sehr unwahrscheinlich. Diese unterscheidet graphisch zwischen 3 h.t und ish.t und damit die zwei Wurzeln ${ }^{11}$ die Assuan-Version jedoch nicht. Der Schreiber von D scheint also anders als der von A auch in dieser Bezeichnung der Göttin stärker an den Sternencharakter der Sothis gedacht zu haben

${ }^{j} N-m$ für $n$ wegen des lautlichen Zusammenfalls von $n$ und $m \cdot{ }^{12}$

${ }^{\mathrm{k}}$ Die Umzeichnung bei Bresciani hat p.t. Brescianis Photo Taf. 22 zeigt jedoch eher $t 3$.

${ }^{1}$ Dieses Epitheton ist im $L G G$ nicht verzeichnet, am nächsten kommt wsr.t n.t nt $r . w \underline{d} r=w$ 'die Mächtigste aller Götter' ${ }^{13}$ und wsr.t $m$ p.t. ${ }^{14}$ das in Dend. II, I 57, 2, eine Bezeichnung der Isis ist. Nach $L G G$ VIII, Ib läge zunächst die Lesung $s^{\top} r^{\urcorner} . t m p$.t h $h r^{\top} n \underline{t} r . w^{\urcorner}$'die am Himmel vor den Göttern verkündet' nahe. Probleme bereitet allerdings, daß am rechten Rand noch Platz für weitere Zeichen wäre, für die die Lesung $s r . t$ vor $m p$.t keine Vorschläge unterbreitet. Die Lösung bietet die D-Parallele, von der aber die hiesige Fassung mit 'Starke in der Erde vor Geb in diesem deinem Namen Starke' offenbar deutlich abweicht, es sei denn-wie einer der anonymen Gutachter des $\mathcal{F E A}$ vorschlägt - in A ist nicht [ 179 zu rekonstruieren sondern und damit ebenfalls 'vor Geb' zu lesen. Das Photo auf Taf. 22 bei Bresciani ließe das zu.

m Die Übersetzung steht im Gegensatz zu 'che signoreggi nel cielo presso gli dèi in quel tuo nome di Signoreggiatrice'. ${ }^{15}$

\footnotetext{
9 Bresciani, in Bresciani und Pernigotti (Hg.), Assuan, 103.

10 Bresciani, in Bresciani und Pernigotti (Hg.), Assuan, ro3.

" Vgl. K. Jansen-Winkeln, “"Horizont” und "Verklärtheit”: Zur Bedeutung der Wurzel 3 ', $S A K$ (I996), $201-I 5$.

I2 Vgl. D. Kurth, Einführung ins Ptolemäische, I (Hützel, 2007), 513, 516.

${ }^{13} L G G$ VIII, 39a

${ }^{14} L G G$ II, $583 \mathrm{a}$

${ }_{15}$ Bresciani, in Bresciani und Pernigotti (Hg.), Assuan, I03.
} 
${ }^{\mathrm{n}}$ Vielleicht auch: 'Thron (s.t) in der Unterwelt vor ...'. Deutliche Abweichung zu D, dort: tn $n . t m$ dws.t hr Wsir $m r n=t$-pfy $n$ T3nn.t 'Erhabene in der Unterwelt vor Osiris in diesem deinem Namen einer Tanenet'.

- Wohl wegen Platzmangels zum Kolumnenende hin ausgelassen.

p Die Namensformel erfordert normalerweise im zweiten Teil ein Epitheton, das durch den ersten Teil aufgrund eines Wortspiels mythologisch bzw. sakramental ausgedeutet wird. Die Lesart von D (vgl. Anm. n) hier zu rekonstruieren, wie das einer der beiden anonymen Gutachter des $\mathscr{F} E A$ vorschlägt, weil der Bereich nach dem Photo auf Brescianis Taf. 22 gänzlich zerstört sei, scheint mir aber nur schwerlich möglich. Denn erstens ist durchaus noch so viel erhalten, daß mir Brescianis Umzeichnung mit $\int_{0}^{\ominus}$ eine plausible, freilich als solche nicht gekennzeichnete Rekonstruktion ist. Zweitens weicht auch der erste, allerdings ebenfalls recht beschädigte Teil der Namensformel in A von der Version D ab. An dieser Stelle kann bereits $\int_{0}^{\circ}$ gestanden haben. Drittens scheint mir der Platz für eine Rekonstruktion entsprechend D zu knapp zu sein, und schließlich ist es viertens in der Namensformel nicht notwendig im zweiten Teil ein Epitheton zu haben, wie die folgenden Beispiele zeigen. In den PT kann statt dessen ein sehr allgemeines 'Gott' stehen: $\underline{t} n-k w$ ini. $n=s n \quad m \quad r n=k n n \underline{t} r$ 'Erhebe dich,' sagen sie, 'in deinem Name eines Gottes. (...)' (Pyr. § I 47b). In Pyr. § $580 \mathrm{~b}$ wird ebenfalls statt eines Namens $n \underline{t} r$ gebraucht und ist dann auch noch mit dem entsprechenden

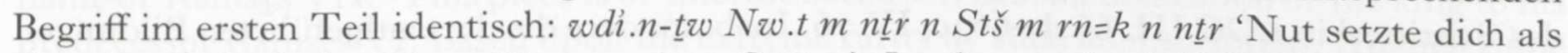
Gott für Seth ein in deinem Namen eines Gottes'. Im Amunsritual (P. Berlin P. 3055) sind gleichfalls die Namen im ersten und zweiten Teil der Namensformel gleich: twt Imn mn m h.t-nb.t $m r n=k n$ 'Imn shm.tw $r n t \underline{t} r . w-n b$.w) 'Du bist Amun, der in allen Dingen dauert, in deinem Namen “Amun”, indem du mächtiger als alle Götter bist' (VII, 5). Die Variante ist noch eintöniger: twt 'Imn $m n$ m h.t-nb〈.t $m r n=k n$ 'Imn 'Du bist Amun, der in allen Dingen dauert, in deinem Namen Amuns' (XIV, 9).

q D: $b 3 h=\underline{t}$ 'du mögest überfluten'. Vielleicht ist das $\underline{t}$ der vermutlich hieratischen Vorlage zu $n=f$ verlesen worden. Die beiden anonymen Gutachter des $\mathscr{F} E A$ wollen sich dem nicht anschließen und schlagen vielmehr vor, $b c h . n=f$ ernstzunehmen und als circumstantiale Form auf die Überschwemmung zu beziehen. Dem stehe ich indes skeptisch gegenüber, denn dann müßte die Überschwemmung wegen des $s \underline{d} m . n=f$ vorzeitig zur Handlung der gepriesenen Göttin sein: '.. die die Überschwemmung ergießt, nachdem sie (= die Überschwemmung) die Beiden Länder überflutet hat'. Tatsächlich kündigt aber der heliakischen Wiederaufgang der Sothis erst die Überschwemmung an, die danach Ägypten überflutet, ${ }^{16}$ d.h. die Ereignisse stehen in einer umgekehrten Reihenfolge, als es aus dem Vorschlag der Gutachter folgte.

${ }^{r}$ Siehe dazu D. Valbelle, Satis et Anoukis (SDAIK 8; Mainz, I98r), I40.

s $\mathrm{D}$ weicht hier $\mathrm{ab}$ und hat: ink $=\underline{t}$ siwur sh.t '... mögest du einleiten die Befruchtung des Feldes'.

${ }_{t-1-t-2} \mathrm{D}$ weicht hier ebenfalls ab und hat $s h p(r . t)$ wnn.t-nb .t) s'nh $h r-n b$ im 'die, die alles, was ist, erschafft, damit jeder davon lebe ...' Leitz, $S A K$ 34, 288, übersetzt hier prospektiv mit eigenständigen Sätzen, was durchaus vertretbar ist. Ich schlage jedoch ein Partizip mit nicht geschriebener Femininendung vor, weil in D das Suffix der zweiten Person Singular femininum mit $=\underline{t}$ oder $=t$ stets eindeutig gekennzeichnet bzw. ausgeschrieben ist, hingegen nur das feminine $t$ ausfallen kann. Das gilt auch für A, wie das in der Transliteration versucht wurde zu zeigen. Insofern scheint mir hier ebenfalls shp $\langle$ r.t $\rangle$, d.h. ein Partizip, zu lesen zu sein. Analog dazu setze ich für die jüngere Bildungsform des Kausativs in A mit di ebenfalls ein Partizip an.

${ }^{16}$ J. F. Quack, 'A Goddess Rising 10,000 Cubits into the Air ... Or Only One Cubit, One Finger?', in J. M. Steele und A. Imhausen (Hg.), Under One Sky: Astronomy and Mathematics in the Ancient Near East (AOAT 297; Münster, 2002), 286; C. Leitz, 'Die Götter, die ihre Majestät begleiten', in D. Kessler, R. Schultz, M. Ullmann, A. Verbovsek, und S. Wimmer (Hg.), Texte-Theben-Tonfragmente:Festschrift für Günter Burkard (ÄAT 76; Wiesbaden, 2009), 308 . 
“ Zur Bedeutung 'untergehen (von der Sonne)' auch des Kausativs shtht, insbesondere in der ptolemäisch-römischen Zeit, siehe schon $W b$. IV, 222.I4. Aufgrund des zur femininen Endung in der vorangehenden Anmerkung Gesagten scheint mir hier $h t p\langle. t\rangle$ zu lesen und mit einem Partizip zu übersetzen zu sein. Daraus folgt, daß in A nicht $s \underline{h t} t \underline{\underline{t}}=\underline{t} \mathrm{zu}$ lesen ist. $\mathrm{Ob}$ es sich um eine Schreibung des Partizips im Femininum handelt oder um den entsprechenden Stativ/Pseudopartizip (.t für .tí als Variante von A im Vergleich zu D mit einem Partizip) möchte ich nicht mit völliger Sicherheit sagen, aber neige zu letzterem, daher auch die obige Übersetzung als Circumstantialis.

$\checkmark$ Das Wort ist mit der Figur der Thoëris geschrieben.

w Quack, Enchoria 27, I го: 'Herrin von Rang in der Barke der Millionen'. Die obige Übersetzung ist als Alternativvorschlag dazu gedacht, der von den Sternen als Mitgliedern der Barkenmannschaft ausgeht. Deren Lauf würde unter der Leitung der Isis-Sothis stehen. ${ }^{17}$ Einer der beiden anonymen $\mathcal{F} E A$-Gutachter weist noch auf $n b n m t . t$ 'freischreitend' hin. Aus inhaltlichen Gesichtspunkten halte ich aber eine solche übertragene Bedeutung für ausgeschlossen, denn im folgenden wird Isis-Sothis als diejenige beschrieben, die die Pläne in der Gottesbarke macht. Beide Prädikationen scheinen mir das Gleiche zu bezeichnen, wie auch davor und danach sich Paare bedeutungsverwandter Epitheta erkennen lassen. Vgl. auch die wörtlichere Übersetzung von $n b$ nmt.t 'Herr des Schrittes' und nb.t nmt.t 'Herrin des Schrittes' in $L G G$ III, 662b-663c, IV, 76. Insbesondere die dort aufgeführten Bildungen mit $m$ p.t lassen daran denken in $n m t . t$ die in $W b$. II, 271.5 nachgewiesene Bedeutung 'vom Wandel der Gestirne' für nmt.t auch hier anzusetzen.

x Vgl. die Diskussion bei Quack, Enchoria 27, I I I.

T3-enh ist entweder der Name verschiedener Nekropolen (der von Esna im dritten oberägyptischen Gau, der von Asyut im dreizehnten oberägyptischen Gau, vielleicht ein Bezirk im zweiten unterägyptischen Gau, vielleicht auch die Nekropole von Heliopolis) oder ein allgemeiner Begriff für die Unterwelt. ${ }^{18}$

Angesichts zweier Versionen desselben Hymnus in Assuan und Dendera stellt sich natürlich die Frage nach der textgeschichtlichen Beziehung beider Fassungen zueinander. A ist unter Ptolemaios IV. (22I-205 v. CHR.) eingemeißelt worden, und D befindet sich auf der Decke des Pronaos von Dendera, die unter Claudius (4I-54 N. CHR.) dekoriert wurde. Das höhere Alter der einen Version ist natürlich kein Argument dafür, sie als Vorlage für die jüngere Fassung anzunehmen, weil A und D auf eine gemeinsame Vorlage zurückgehen mögen. Dennoch möchte ich vorschlagen, daß A ein Indiz dafür ist, die Entstehung des Hymnus im Umfeld von Syene/Elephantine annehmen zu dürfen. Denn die Bedeutung Elephantines in der ägyptischen Vorstellungswelt als Ursprungsort der Nilflut bzw. dem Ort Ägyptens, den die Überschwemmung als erstes erreicht, ${ }^{19}$ paßt zur Preisung der Isis-Sothis als Bringerin der Nilflut in dem hier besprochenen Hymnus. Das erhöht die Wahrscheinlichkeit, zu Recht in A einen Textzeugen zu erkennen, der nicht allzu weit von dem Ort erhalten geblieben ist, an dem er erstmals verfaßt wurde. Auch wenn die dritte Kolumne auf dem Türrahmen des Isis-Tempels von Assuan mehr oder weniger eigenständig ist und hier Isis kosmisch-universalistisch geschildert wird, so können die darin erwähnten astronomischen Kompetenzen, ihre Zuständigkeit für die Nekropole $\left(T_{3-}-n h\right)$ und die Bezüge nach Heliopolis (Isis als Herrin im Beamtenhaus und Einzige im Phönixhaus) Reprisen dieser Nilflutthematik

17 Vgl. M. A. Stadler, Weiser und Wesir: Studien zu Vorkommen, Rolle und Wesen des Gottes Thot im ägyptischen Totenbuch (Orientalische Religionen in der Antike I; Tübingen, 2009), 442.

${ }^{18}$ H. Gauthier, Dictionnaire des noms géographiques, VI (Cairo, I93I), 6. Siehe auch M. Smith, The Liturgy of Opening the Mouth for Breathing (Oxford, I993), 64.

${ }_{19}$ H. Jaritz, Elephantine, III: Die Terrassen vor den Tempeln des Chnum und der Satet. Architektur und Deutung (AV 32; Mainz am Rhein, I980), 64; J. F. Pécoil, 'Les sources mythiques du Nil et le cycle de la crue', BSEG I7 (1993), 97-I го. Vgl. z.B. auch den Tagwählkalender: am IV. 3h.t r bringt, heißt es dort, einen Erlaß Res nach Süden (d.h. Elephantine), um Hapi den erreichten Idealzustand der Überschwemmung zur Kenntnis zu bringen; siehe C. Leitz, Tagewählerei: Das Buch ḩst nhh ph.wy d़ t und verwandte Texte (ÄA 55; Wiesbaden, I994), I47-9. 
sein: die durch das Sirius-Gestirn angekündigte Nilflut als Ausfluß des Osiris, dessen Grab in Bigge als Nilquelle galt, und Heliopolis als ein anderer Ort Ägyptens, an dem ebenfalls eine Nilquelle lokalisiert wurde. ${ }^{20}$ Die dritte Kolumne, die sich an A anschließt, deprovinzialisiert damit Isis' Kompetenzen in Bezug auf den Nilstand. Und so ist es nicht überraschend, wie wenig das in dem Hymnus anklingende Lokalkolorit daran hinderte, auch an anderen Orten in Ägypten, etwa in Dendera, den Text zu schätzen.

Martin Andreas Stadler 